\title{
Subcutaneous Sarcoidosis Occurring in Both Chin and Toe
}

\author{
Kwang Seog Kim, \\ Dong Seob Lim, \\ Jun Ho Choi, \\ Jae Ha Hwang, \\ Sam Yong Lee
}

Department of Plastic and Reconstructive Surgery, Chonnam National University

Medical School, Gwangju, Korea
No potential conflict of interest relevant to this article was reported.
Sarcoidosis is a systemic inflammatory disease characterized by non-caseating granulomas of unknown origin. Of the fewer than $6 \%$ of sarcoidosis cases that occur in subcutaneous tissue, most occur on the face or forearm, but rarely in the toe. A 33-year-old man was admitted to our institute with a 2-cm mass on his chin and a $0.5-\mathrm{cm}$ mass on his right fourth toe. Based on preoperative ultrasonography, epidermal cysts were suspected, and histopathological tests were performed after removing the masses. Histopathologically, the chin and toe tissue samples showed chronic granulomatous inflammation, without necrosis, indicative of sarcoidosis. Chest computed tomography revealed a large number of small nodules in both the interlobar fissures and the peribronchial area, and a large number of small lymph nodes in both the hilar and interlobar node areas. On the basis of the histopathologic and imaging findings, the patient was diagnosed with sarcoidosis. This very rare case of sarcoidosis, occurring in both chin and toe, suggests that thorough evaluation is needed to find other mass when we find sarcoidosis in facial area.

Keywords: Face / Toes / Subcutaneous tissue / Sarcoidosis

\section{INTRODUCTION}

Sarcoidosis is a systemic inflammatory disease characterized by non-caseating granulomas of unknown origin. The disease may occur in people regardless of their ethnicity, sex, or age; it is prevalent among individuals in their 20s and 30s, but is rare among Asians [1]. Fewer than $6 \%$ of sarcoidosis cases involve the subcutaneous tissue. When it does, it usually occurs on the face or forearm; to date, only a single case of sarcoidosis involving the subcutaneous tissue of a toe has been reported [2]. We describe a case of sarcoidosis involving the lungs and subcutaneous tissue of the chin and a toe.

\footnotetext{
Correspondence: Kwang Seog Kim

Department of Plastic and Reconstructive Surgery, Chonnam National University Medical School, 42 Jebong-ro, Dong-gu, Gwangju 61469, Korea

E-mail: pskim@chonnam.ac.kr

Received August 24, 2017 / Revised September 5, 2017 / Accepted September 7, 2017
}

\section{CASE REPORT}

A 33-year-old man was admitted to our institute with a 2-cm mass on his chin and a $0.5-\mathrm{cm}$ mass on his right fourth toe (Fig. 1). The patient had discovered the masses 1 month prior to admission, but did not experience tenderness around the masses. Preoperative ultrasonographic findings were indicative of epidermal cysts (Fig. 2), and the masses were excised under local anesthesia. The masses, with ambiguous boundaries, showed firm adhesion to nearby tissues, spanning the subcutaneous and adipose tissue layers (Fig. 3). Post-excisional histopathology of the masses demonstrated chronic granulomatous inflammation, without necrosis, indicative of sarcoidosis (Fig. 4). Despite the absence of respiratory symptoms, the patient underwent chest computed tomography (CT) because sarcoidosis frequently affects the lungs. The chest CT results revealed a large number of small nodules in both the interlobar fissures and peribronchial area, and a large number of small 

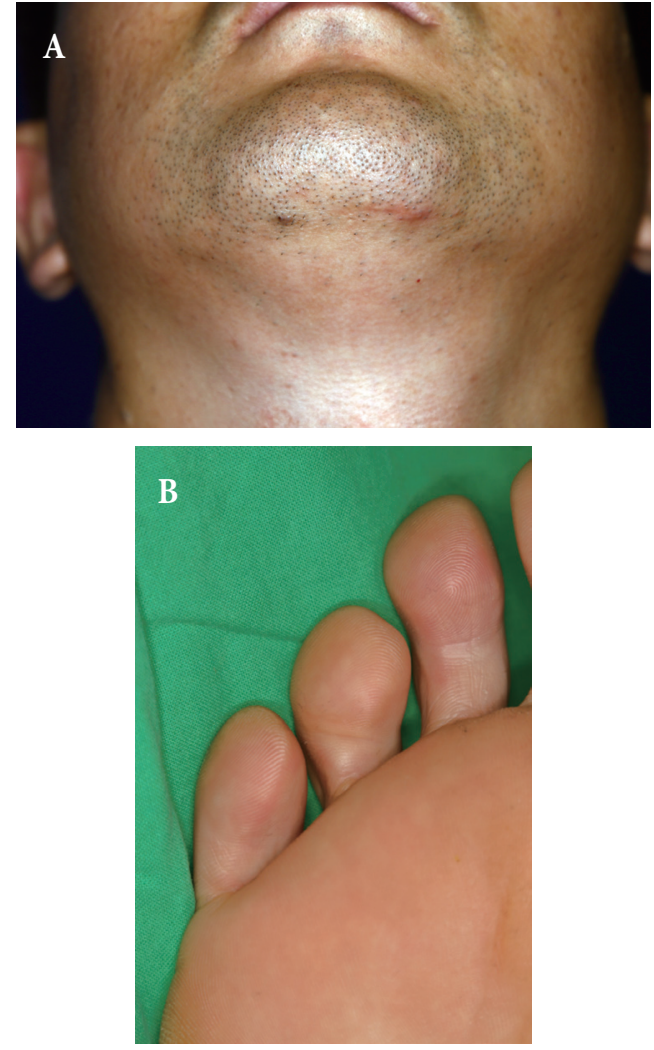

Fig. 1. (A, B) Preoperative views showing subcutaneous masses in the chin and right fourth toe.

lymph nodes in both the hilar and interlobar nodal areas (Fig. 5). On the basis of the histopathologic and imaging results, the patient was diagnosed with sarcoidosis.

\section{DISCUSSION}

Masses occurring on the toe include those arising from fibromas, giant cell tumors, pigmented villonodular synovitis, synovial sarcomas, digital fibromatosis, granuloma annulare, septic arthritis, and inflammatory arthropathy. However, sarcoidosis has rarely been described as the cause of masses involving the lower digits.

Histologically, cutaneous sarcoidosis is divided into specific and non-specific granulomatous lesions [3]. Subcutaneous sarcoidosis, belonging to the specific lesion group, usually occurs on the face, forearm, and lower leg, accounting for approximately $1.4 \%-6.0 \%$ of all sarcoidosis cases. The lesions rarely occur on the
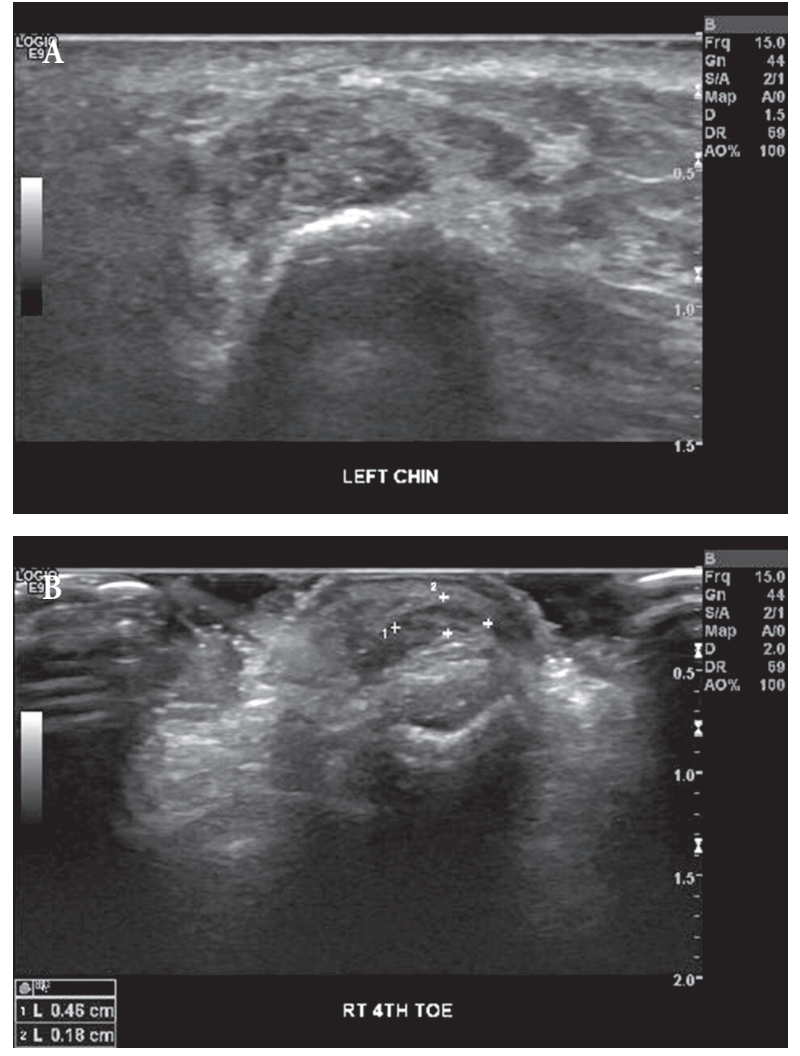

Fig. 2. Ultrasonogram of the chin and right fourth toe. (A) A $1.93 \times 0.71-\mathrm{cm}$, lobulated, hypoechoic lesion in the subcutaneous layer of the left chin, initially believed to be an epidermoid cyst. (B) A $0.5 \times 0.2-\mathrm{cm}$, well-defined, hypoechoic lesion in the subcutaneous layer of the right fourth toe, initially believed to be a benign lesion.

fingers or toes, with the only known diagnosis of digital subcutaneous sarcoidosis having been reported [2].

Sonographically, the non-caseating granuloma associated with subcutaneous sarcoidosis is characteristically more hypoechoic than the nearby inflamed tissues. Although such sonographic findings may also be seen in patients with tuberculosis, they can be helpful for the early diagnosis of subcutaneous sarcoidosis. On T2weighted magnetic resonance images, lesions with indistinct boundaries are observed in the subcutaneous tissue, while the proton-weighted images characteristically show a mid-level signal [4].

Differential diagnosis of sarcoidosis involves eliminating other diseases that can show similar clinical symptoms, e.g., coughs; shortness of breath; chest discomfort; and the formation of red, bumpy lesions through the skin, in addition to histopathological 

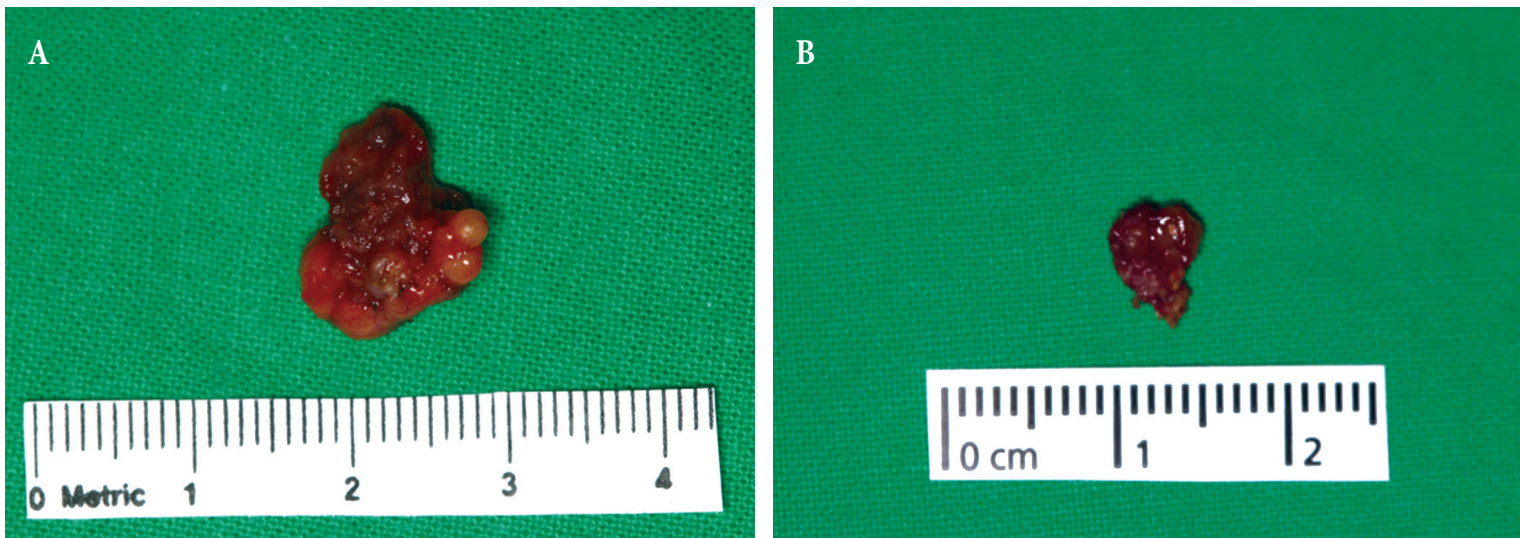

Fig. 3. Macroscopic appearance of the excised masses from the (A) chin and (B) right fourth toe.
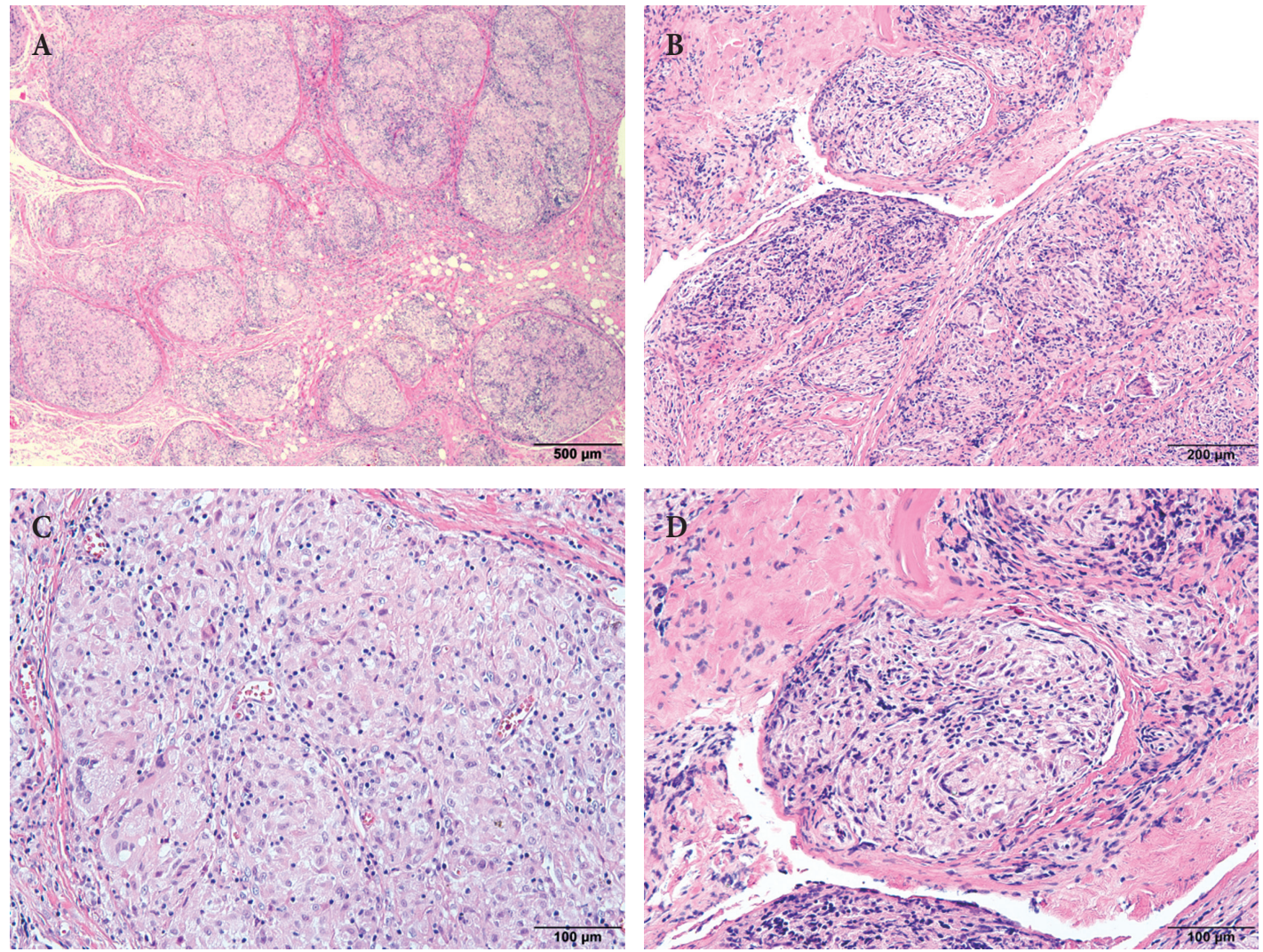

Fig. 4. Histopathologic images of subcutaneous sarcoidosis. Lesions from the (A) chin and (B) right fourth toe showing discrete multiple granulomata, without caseous necrosis $(\mathrm{H} \& \mathrm{E}, \times 40)$. Granuloma, from the $(\mathrm{C})$ chin and $(\mathrm{D})$ right fourth toe, composed of epithelioid histiocytes, with abundant cytoplasm, and Langerhans' giant cells $(\mathrm{H} \& \mathrm{E}, \times 200)$.

evidence of non-caseating granuloma [5]. In our case, the mass lesions on the chin and toe were histopathologically identified as a non-caseating granuloma, with the diagnosis of early sarcoidosis being made based on the addition of chest CT results and clinical symptoms.

Depending on the originating organ and the range of involve- 


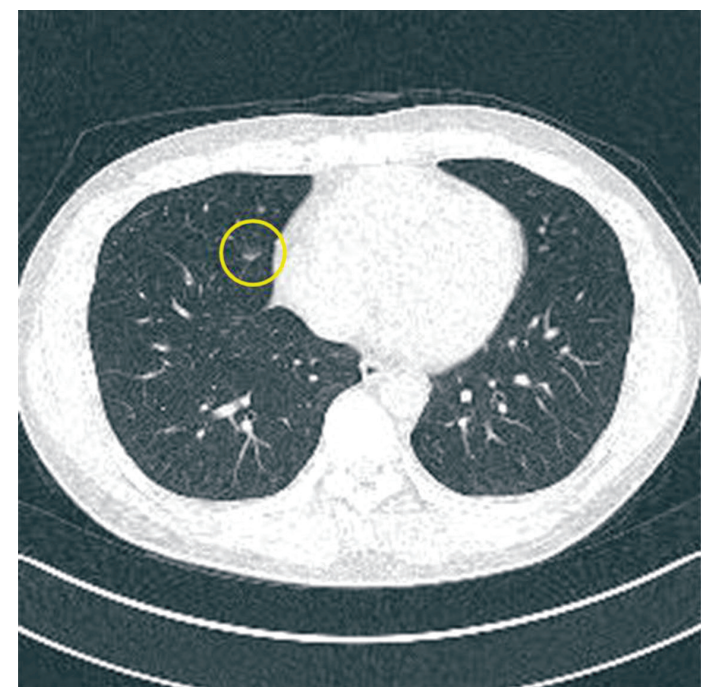

Fig. 5. Chest computed tomography. There are multiple small nodules along both the interlobar fissures and peribronchial areas in the right lower lobe (yellow cycle) and in the lingular segment of the left upper lobe, suggestive of early parenchymal involvement of the sarcoidosis. There are several small to borderline-enlarged lymph nodes in both the hilar and interlobar nodal areas.

ment, sarcoidosis is a disease with a range of outcomes, from cases that resolve spontaneously to cases that are fatal due to complications associated with respiratory failure or involvement of the heart or nervous system. Treatment typically involves regulating the level of inflammation by administering steroids or immunosuppressants, such as prednisolone.

In cases of sarcoidosis involving the skin, aesthetic problems may also serve as indicators of treatment. However, when early sarcoidosis occurs as a subcutaneous mass, a biopsy-based diagnosis is essential. In this case, the patient was diagnosed with early sarcoidosis, and no specific findings or signs of organ failure were observed during the general examination. Therefore, observation, rather than immunosuppressive treatment, was initiated. However, the patient was educated to have an early awareness of possible exacerbations and to prevent disease progression through longterm follow-up of his other organs, including the eyes.

Because sarcoidosis involving the subcutaneous tissue layer of a toe is extremely rare and because it can serve as an important sign for the diagnosis of systemic sarcoidosis, its clinical implication is significant. The findings from this case suggest that thorough evaluation is needed to find other mass when we find sarcoidosis in facial area.

\section{PATIENT CONSENT}

The patient provided written informed consent for the publication and the use of their images.

\section{REFERENCES}

1. Lodha S, Sanchez M, Prystowsky S. Sarcoidosis of the skin: a review for the pulmonologist. Chest 2009;136:583-96.

2. Celik G, Ciledag A, Akin P, Simsek Y, Kaya A, Numanoglu N, et al. Subcutaneous sarcoidosis with plantar involvement. Ann Dermatol 2010;22:435-8.

3. Ali MM, Atwan AA, Gonzalez ML. Cutaneous sarcoidosis: updates in the pathogenesis. J Eur Acad Dermatol Venereol 2010;24:747-55.

4. Chen HH, Chen YM, Lan HH, Lee CH, Chen DY. Sonographic appearance of subcutaneous sarcoidosis. J Ultrasound Med 2009;28:813-6.

5. Kim YW. Sarcoidosis. J Korean Med Assoc 2009;52:41-8. 